\title{
Integration of InP/InGaAs/InP $p$-i-n photodiodes on silicon via wafer bonding and hydrogen-induced layer exfoliation
}

\author{
Peng Chen ${ }^{1}$, Ka Ming Wong ${ }^{1}$, Kei May Lau ${ }^{1}$, and S. S. Lau ${ }^{2}$ \\ ${ }^{1}$ Department of Electronic and Computer Engineering, Hong Kong University of Science and Technology, Hong Kong, \\ China \\ Phone: +(852)23585033, Fax: +(852)23581485, Email: eepchen@ust.hk \\ ${ }^{2}$ PDepartment of Electrical and Computer Engineering, University of California, San Diego, La Jolla, California, USA
}

\begin{abstract}
Functioning InP/InGaAs/InP p-i-n photodiodes, initially grown on an InP substrate, were transferred onto a $\mathrm{SiO}_{2} / \mathrm{Si}$ substrate using the combination of ion-cutting and selective chemical etching. Effects of hydrogeninduced defects on transferred devices are discussed.

\section{Introduction}

The integration of III-V compounds with silicon substrate is motivated by the requirements for optoelectronic integrated circuits (OEIC) and silicon photonics [1-2]. This type of heterogeneous integration enables the combination of III-V based optoelectronic devices with Si-based microelectronic circuits. Wafer bonding technique has been widely used to transfer III-V thin films onto Si. In the case of ion-cutting (or Smart CutTM) process based on wafer bonding and hydrogeninduced layer exfoliation, it is common to transfer III-V layers onto Si. The transferred structure is then used as a template for subsequent epitaxial re-growth of device structures [3-5]. Due to the rough as-transferred surface and considerable implantation-induced radiation damage near the surface region of transferred structure, a chemical mechanical polishing (CMP) process and/or other surface treatments are required before re-growth. Without complete damage removal, the re-grown III-V layers often suffer from the propagation of remaining radiation damage in the transferred layers. In this work, we studied an alternative approach in which a device layer structure is transferred onto Si substrate followed by standard device fabrication processes, and thus no further re-growth is required.
\end{abstract}

\section{Experiments}

Figure 1 shows the entire process flow and layer structures used in this work. A $p$-InP $(140 \mathrm{~nm}) / i$ $\operatorname{InGaAs}(260 \mathrm{~nm}) / n-\operatorname{InP}(150 \mathrm{~nm}) / \operatorname{InGaAs}(300 \mathrm{~nm}) / \mathrm{InP}$ heterostructure was grown by metal organic chemical vapor deposition (MOCVD) on the $n$-type InP (100) wafer. The top $p$-InP layer was doped in-situ with $\mathrm{Zn}$ to a concentration of $2 \times 10^{18} \mathrm{~cm}^{-3}$ and the $n$-InP layer was doped with $\mathrm{Si}$ to $3 \times 10^{18} \mathrm{~cm}^{-3}$. $\mathrm{H}^{+}$ions were implanted at $160 \mathrm{keV}$ with a dose of $8.5 \times 10^{16} \mathrm{~cm}^{-2}$. After implantation the protective SiNx layer was chemically removed. A piece of implanted sample and a piece of Si substrate, which had been coated with a 68 nm-thick thermal $\mathrm{SiO}_{2}$ layer, were cleaned with organic solutions (TCE, Acetone, and IPA in sequence) and RCA1 solution $\left(\mathrm{NH}_{4} \mathrm{OH}: \mathrm{H}_{2} \mathrm{O}_{2}: \mathrm{H}_{2} \mathrm{O}=1: 2: 10\right)$. After an oxygen plasma activation, the two pieces were bonded in air at room temperature, followed by a thermal anneal at $90{ }^{\circ} \mathrm{C}$ for 10 hours to increase the bonding strength. Upon a subsequent annealing at $140{ }^{\circ} \mathrm{C}$, the bonded structure exfoliated around the depth of the projected range of hydrogen in the original InP substrate. The transferred structure was further annealed up to $650{ }^{\circ} \mathrm{C}$ in $\sim 10 \%$ $\mathrm{H}_{2} / \mathrm{N}_{2}$ forming gas for 15 minutes, and the top InP and InGaAs layers were selectively etched, resulting in an $\mathrm{InP} / \mathrm{InGaAs} / \mathrm{InP} n-i-p$ structure on the $\mathrm{SiO}_{2} / \mathrm{Si}$ substrate for final photodiodes fabrication. $p$-i-n photodiodes were also fabricated on the as-grown sample as a control sample. To ascertain the effect of hydrogen ion implantation damage on device performance, $p-i-n$ photodiodes were fabricated directly on the $\mathrm{H}^{+}$asimplanted sample (see Figure 1(b)) using identical fabrication process as on the as-grown sample.

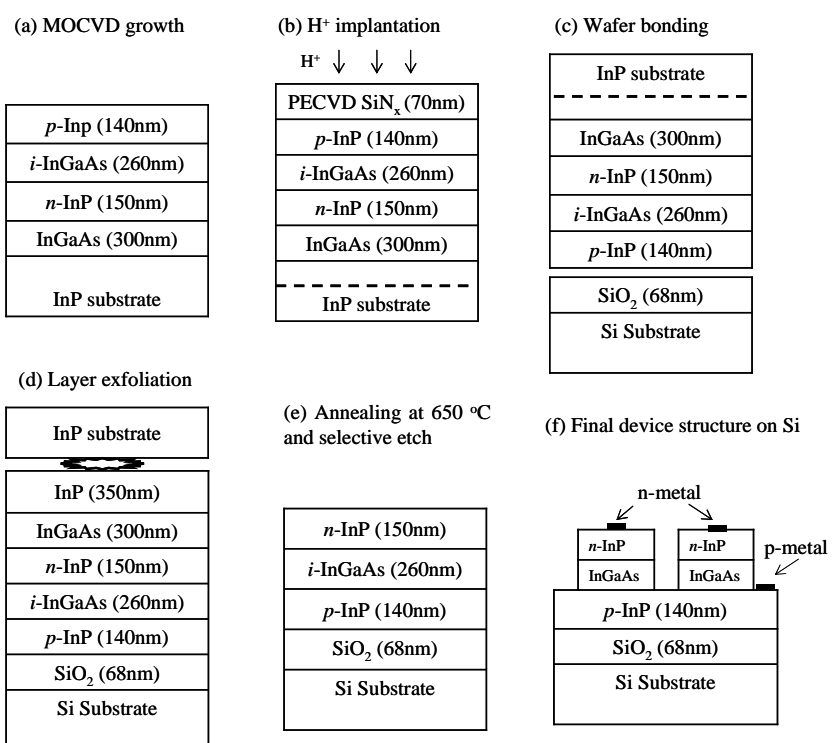

Figure 1: Schematic drawing of the process flow for transferring a p-i-n photodiode onto $\mathrm{Si}$, based on ioncutting and selective chemical etching.

\section{Results and discussions}

$\mathrm{I}-\mathrm{V}$ curves from as-grown and hydrogen as-implanted photodiodes are presented in Figure 2, respectively. The current limit was set as $0.01 \mathrm{~A}$ during the measurements. The photodiode shows a typical p-n junction behavior in Figure 2 (a), with a turn-on voltage of $\sim 0.85 \mathrm{v}$ and a 
breakdown voltage of $\sim 3.7 \mathrm{v}$. It is obvious that after hydrogen implantation, the p-i-n photodiodes exhibited a short-circuit element-like I-V behavior. Since at this asimplanted stage before any annealing steps, all hydrogen ions were inside the sample and all the implantation induced radiation damage was also in the sample. The presence of the high-density defects in the implanted sample enabled carrier hopping through the whole $p-i-n$ structure [6], resulting in a resistor-like behavior.

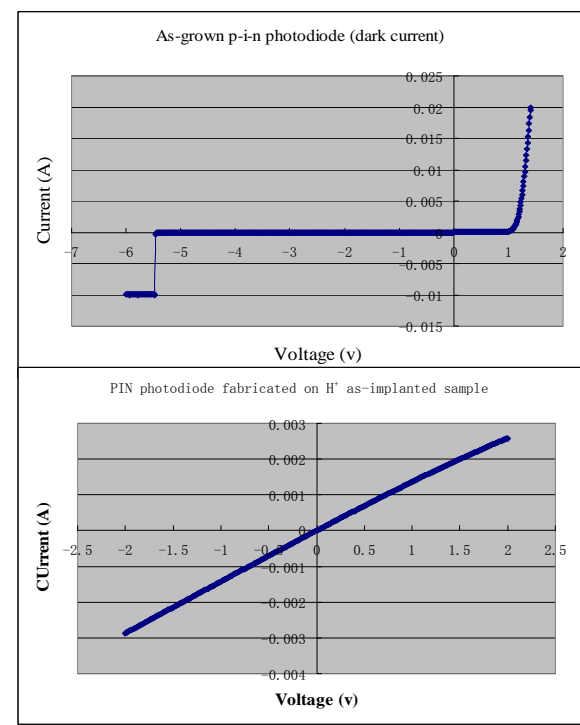

Figure 2: Dark current of the p-i-n photodiodes made on: (a) as-grown sample; (b) hydrogen as-implanted sample.
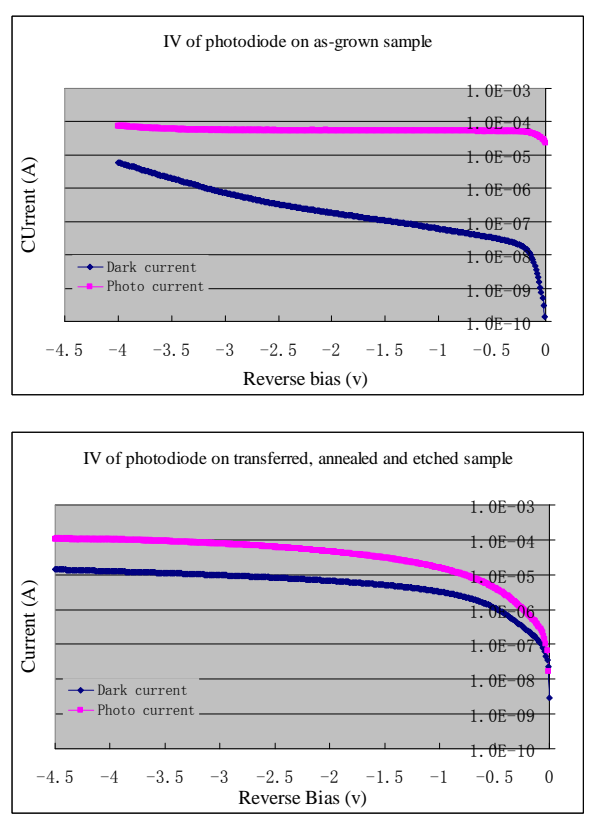

Figure 3: Dark current (blue line) and photo current (pink line) in the reverse biased region: (a) as-grown p-i$\mathrm{n}$ photodiodes on InP sub; (b) after transfer onto $\mathrm{Si}$ followed by annealing at $650{ }^{\circ} \mathrm{C}$ and selective etching.

For the as-grown sample and final transferred and annealed sample, the photo current was measured with an infrared light source at a wavelength of $1.55 \mu \mathrm{m}$ and a power of $2 \mathrm{mw}$, as shown in figure 3. For the transferred photodiodes on $\mathrm{Si}$, the dark current was $\sim 1.5$ orders higher than the as-grown sample and the photo current was comparable to the as-grown sample. The photo current became a function of the applied field.

The degradation of the electrical properties due to the damage induced by hydrogen implantation in InP has been demonstrated in the literature and also by this group. The antisite defects $\left(\mathrm{In}_{\mathrm{P}}\right.$ and $\left.\mathrm{P}_{\mathrm{In}}\right)$ and related defect complexes created in the replacement collisions are believed to act as the deep level carrier trapping centers, where $\operatorname{In}_{\mathrm{P}}$ is responsible for electron trapping and $\mathrm{P}_{\mathrm{In}}$ for the hole trapping [7]. Annealing could recover some of the defects but there was still considerable damage in the final transferred structure. These observations suggest that the recovery of the implantation-induced damage determines to a large extent the performance of minority carrier devices.

\section{Conclusions}

This work provides direct evidence of the effects of hydrogen implantation-induced damage, especially the antisite defects, on the performances of transferred minority carrier devices. By annealing at $650{ }^{\circ} \mathrm{C}$ for 15 min combined with selective etch after transfer, much of the implantation damage in the $n$-type and undoped layers was removed. However, substantial degradation of the current-voltage characteristics including a much higher dark current was observed due to the defect residues in $p$-type InP layer. Further optimization of the process control and device structure design is required.

\section{References}

1. J. W. Goodman, F. J. Leonberger, S.-Y. Kung, and R. A. Athale, Proc. IEEE 72, (1984), p850.

2. H. Wada, H. Sasaki, and T. Kamijoh, Solid-State Electron. 43, (1999), p1655.

3. S. Hayashi, R. Sandhu, M. Wojtowicz, G. Chen, R. Hicks, and M. S. Goorsky, J. Appl. Phys. 98, (2005), p093526.

4. J. M. Zahler, K. Tanabe, C. Ladous, T. Pinnington, F. D. Newman, and H.A. Atwater, Appl. Phys. Lett. 91, (2007), p012108.

5. S. Hayashi, R. Sandhu, and M. S. Goorsky, J. Electrochem. Soc. 154, (2007), H293.

6. S. J. Pearton, C. R. Abernathy, M. B. Panish, R. A. Hamm, and L. M. Lunardi, "Implant-induced highresistivity regions in InP and InGaAs”, J. Appl. Phys. 66, (1989), p656.

7. S. J. Pearton, "Ion implantation for isolation of III-V semiconductors”, Mater. Sci. Rep. 4, (1990), p313. 